\title{
Soluble P-tau217 reflects both amyloid and tau pathology in the human brain and mediates the association of amyloid with neocortical tau
}

Niklas Mattsson-Carlgren ( $\nabla$ niklas.mattsson@med.lu.se )

Lund University

Shorena Janelidze

Lund University

Randall Bateman

Department of Neurology, Washington University School of Medicine

Ruben Smith

Lund University

Erik Stomrud

Lund University

Geidy Serrano

Banner Alzheimer's Institute

Eric Reiman

Banner Alzheimer's Institute

Sebastian Palmqvist

Lund University https://orcid.org/0000-0002-9267-1930

Jeffrey Dage

Eli Lilly and Company

Thomas Beach

Banner Sun Health Research Institute

Oskar Hansson

Lund University https://orcid.org/0000-0001-8467-7286

\section{Article}

Keywords: Alzheimer's disease, Plasma Levels, Post-mortem Neuropathological Data, PET Imaging

Posted Date: November 13th, 2020

DOI: https://doi.org/10.21203/rs.3.rs-101153/v1 
License: (c) (i) This work is licensed under a Creative Commons Attribution 4.0 International License. Read Full License 


\section{Abstract}

Alzheimer's disease is characterized by $\beta$-amyloid plaques and tau tangles. Plasma levels of phosphotau217 (P-tau217) accurately differentiate Alzheimer's disease dementia from other dementias, but it is unclear to what degree this reflects $\beta$-amyloid plaque accumulation, tau tangle accumulation, or both. In a cohort with post-mortem neuropathological data $(\mathrm{N}=88)$, both plaque and tangle density contributed independently to higher P-tau217. Several findings were replicated in a cohort with PET imaging ("BioFINDER-2", N=426), where $\beta$-amyloid and tau PET were independently associated to P-tau217. Ptau 217 correlated with $\beta$-amyloid PET (but not tau PET) in early disease stages, and with both $\beta$-amyloid and (more strongly) tau PET in late disease stages. Finally, P-tau217 mediated the association between $\beta$ amyloid and tau in both cohorts, especially for tau outside of the medial temporal lobe. These findings support the hypothesis that plasma P-tau217 is increased by both $\beta$-amyloid plaques and tau tangles and is congruent with the hypothesis that P-tau is involved in $\beta$-amyloid-dependent formation of neocortical tau tangles.

\section{Introduction}

$\beta$-amyloid (AB) pathology and tau pathology are core hallmarks of Alzheimer's disease (AD). The amyloid cascade hypothesis states that altered $A \beta$ metabolism is an initiating event, which drives accumulation of aggregated tau ${ }^{1}$. These events are associated with altered levels of fluid biomarkers, as has been extensively studied in both cerebrospinal fluid (CSF) and plasma2,3. Recently, it has been shown that plasma levels of hyperphosphorylated tau (including both P-tau $181^{4,5}$ and P-tau $217^{6}$ ) are markedly elevated in patients with AD. Plasma P-tau217 levels have particularly high accuracy for AD, and are correlated with tangle densities in post-mortem data ${ }^{6}$. However, recent studies have suggested that CSF P-tau217 (and other P-tau variants) can be increased as a function of $A \beta$ accumulation, in individuals who are tau negative as determined by positron emission tomography $(\mathrm{PET})^{7,8}$ (but note that tau PET has limited sensitivity to detect very sparse tau aggregation ${ }^{9}$ ). It is unclear to what degree plasma P-tau217 levels depend on $A \beta$ versus tau load in the brain. Here, we set out to clarify this question in two cohorts, including a cohort with post-mortem quantification of $A \beta$ plaques and tau tangles, and a large prospective cohort where $A \beta$ and tau load were measured in vivo using PET imaging. In sum, we found converging evidence from both cohorts that $A \beta$ and tau load had independent and interactive effects on plasma P-tau217. We also found that plasma P-tau217 levels mediated the effect of A $\beta$ load on tau load. Finally, we found that in subjects with very limited tangle pathology, $A \beta$ plaque load but not medial temporal lobe tangle load remained statistically associated with plasma P-tau217. Taken together, this supports the theory that the first increases in release and phosphorylation of soluble tau measured in plasma are driven by $A B$ aggregation and may appear largely before spread of tau tangles outside of the medial temporal lobe. One possibility is that soluble P-tau217 plays a role in the spread of neocortical tangles, which could motivate trials with treatments targeting soluble phosphorylated tau, to break the link between $A \beta$ and tau aggregation in $A D$. 


\section{Results}

\section{Plasma P-tau217 is independently associated with pathology measures of both $A \beta$ and tau}

We first tested associations between antemortem plasma P-tau217 and neuropathological quantification of $A \beta$ plaques and tau tangles in a cohort with post-mortem data $(N=88)$. We compared regression models with plasma P-tau217 as response variable and different sets of predictors, including I) only $A \beta$ plaque density, II) only tau tangle density, III) both plaque and tangle density, IV) plaque and tangle density and their interaction. All models included age and sex as covariates. For the individual models, the tangle only model had slightly higher overall explanatory power $\left(R^{2}=0.47\right)$ than the plaque only model $\left(R^{2}=0.40\right)\left(\triangle A I C=9\right.$ favoring the tangle only model, but the difference in $R^{2}$ was not significant, tested in a bootstrap procedure, $\Delta \mathrm{R}^{2}=0.07,95 \% \mathrm{Cl}-0.17$ to 0.25 ). Model comparisons favored including both plaques and tangle densities in an interaction model (Fig. 1A, $\triangle \mathrm{AIC}=5-29$ compared to the other models). In the interaction model, both plaque density $(\beta=2.00, P<0.0001$; compared to $\beta=2.95, P<$ 0.0001 in the plaque only model), tangle density $(\beta=1.46, P=0.0072$; compared to $\beta=3.29, P<0.0001$ in the tangle only model) and the plaque by tangle interaction $(\beta=1.26, P=0.012)$ were significantly associated with higher plasma $P$-tau217 levels. Age $(P=0.19)$ and sex $(P=0.59)$ were non-significant in the interaction model. Higher plaque density had the strongest association with high plasma P-tau217 among those with higher tangle density (visualized with tertiles of tangles in Fig. 1B), and higher tangle density had the strongest association with high plasma P-tau217 among those with higher plaque density (visualized with tertiles of plaques in Fig. 1C). For all these analyses, plaque and tangle densities were used as continuous variables, centered and scaled to z-scores. These results supported the hypothesis that plasma P-tau217 is increased by independent effects of both $A \beta$ and tau pathology.

\section{Plasma P-tau217 mediates the effect of $\mathrm{A} \beta$ on tau}

We next conducted mediation analyses in the neuropathology cohort (Fig. 2). Plasma P-tau217 partly mediated the effect of $A \beta$ plaque density on tau tangle density (but $A \beta$ plaque density retained a significant association with tangle density also in the presence of plasma P-tau217) (60\% mediation, Fig. 2A). In contrast, there was no significant mediation of effects of $A \beta$ plaque density on tau tangle density in the medial temporal lobe (entorhinal cortex and hippocampus) (the direct effect of $A \beta$ plaques on tau tangles was also weaker, Fig. 2B). The strongest mediation was seen for total tangle density when removing tangles in the medial temporal lobe ( $77 \%$ mediation; the direct effect of $A \beta$ plaques on tangles became non-significant, Fig. 2C). These results showed that plasma P-tau217 may partly explain the link between build-up of $A \beta$ and tau pathology, especially for tau pathology that extends over the neocortex, beyond the medial temporal lobe.

\section{No associations between plasma P-tau217 and isolated medial temporal lobe tau}


To study the effects of very subtle tau pathology on plasma P-tau217, we did a subset analysis on the neuropathology cohort participants without tangles in the parietal or frontal lobe (and only minimal tangle pathology in the temporal lobe, allowing score 0.5 , indicating a density halfway between "none" [0] and "sparse" [1])). In this subset $(\mathrm{N}=42), A \beta$ plaque density remained significantly associated with plasma $P$-tau217 ( $\beta=1.64, P<0.0001$ ) (Fig. 3A), but medial temporal lobe tangles were not associated with plasma $P$-tau217 $(P=0.39$, Fig. $3 B$ ) and there was no association between $A \beta$ plaque density and medial temporal lobe tangle density $(P=0.59, \mathrm{Fig} .3 C)$. These results show that $A \beta$ pathology is linked to increased phosphorylation and/or release of tau even in very early stages of $A D$ (without prominent tangle pathology in the neocortex). In contrast, tau pathology which is contained in the medial temporal lobe without significant neocortical involvement may occur independently of $A \beta$ pathology and without altered phosphorylation or release of soluble tau.

\section{Plasma P-tau217 is independently associated with PET measures of both $A \beta$ and tau}

We also tested associations between plasma P-tau and measures of aggregated $A \beta$ and tau in vivo in the BioFINDER-2 cohorts (see Table 1). We again compared regression models with plasma P-tau217 as response variable and different sets of predictors, including I) only A $\beta$ PET (using a large cortical regionof-interest [ROI]), II) only tau PET (using a global cortical ROI representing Braak stages I-VI), III) both $A \beta$ PET and tau PET, IV) A $\beta$ PET and tau PET and their interaction. Model comparison of adjusted $\mathrm{R}^{2}$ and AIC favored the model with the interaction term over the other models (Fig. $4 \mathrm{~A}$ ). In the interaction model, both A $\beta$ PET $(\beta=1.08, P<0.0001)$, tau PET $(\beta=0.43, P<0.0001)$ and the $A \beta$ by tau PET interaction $(\beta=0.31, P$ $<0.0001$ ) were significantly associated with greater plasma P-tau217 ( $A \beta$ and tau PET were used as continuous variables, centered and scaled to $z$-scores $)$. Age $(P=0.86)$ and sex $(P=0.94)$ were nonsignificant predictors in the model. Higher A $\beta$ PET was associated with higher plasma P-tau217 at all levels of tau PET (with strongest association in those with highest tau PET) (Fig. 4B). Higher tau PET was associated with higher plasma P-tau217 in those with high (but not in those with low) A $\beta$ PET (Fig. 4C). Figure 5 shows another representation of these independent effects of $A \beta$ PET and tau PET, with Ptau217 levels for different combination of amyloid PET and tau PET tertiles. The highest tertile of A $\beta$ PET had higher plasma P-tau217 compared to low/average A $\beta$ PET, at all levels of tau PET. In contrast, the highest tertile of tau PET uptake was associated with higher plasma P-tau217 mainly in the highest A $\beta$ PET tertile (rather than in those with low/average A $\beta$ PET). These results largely confirmed our results from the neuropathology dataset, showing that plasma P-tau217 is independently related to both $A \beta$ and tau build-up. 
positivity, plasma P-tau217 correlated with A PET (R2 = 0.10) but not tau PET (Fig. 6B and 6E). In the group positive for both $A \beta$ and tau, plasma P-tau217 correlated with both $A \beta$ PET and tau PET but had strongest correlation with tau PET ( $R 2=0.46$ for tau, $R 2=0.14$ for $A \beta$ ). This supported our hypothesis that plasma P-tau217 reflects both $A \beta$ and tau accumulation in $A D$, but primarily reflects $A \beta$ accumulation during the early disease stages, and primarily tau accumulation during the later disease stages.

\section{Plasma P-tau217 mediates effects of A PET on tau PET}

We also conducted mediation analyses to test to what degree plasma P-tau217 explained effects of $A \beta$ aggregation on tau uptake in BioFINDER-2. Plasma P-tau217 partly mediated the effect of A $\beta$ PET on global tau PET (63\% mediation, Fig. 7A). The mediation was attenuated for tau PET in the medial temporal lobe (entorhinal cortex and hippocampus, $29 \%$, Fig. 7B). This is consistent with the hypothesis that the mechanism that links $A \beta$ build-up to tau aggregation involves increased phosphorylation and/or release of soluble tau, and that this is especially important for tau aggregation that occurs outside of the medial temporal lobe (entorhinal cortex and hippocampus).

\section{Discussion}

With recent advances in assay development, it is now possible to measure plasma levels of P-tau217 in clinical research, drug trials, and perhaps also in clinical practice in the future. To best interpret the Ptau217 results it is important to understand what plasma P-tau217 levels represent. Although proposed frameworks have largely assumed that soluble P-tau best mirrors tau deposition ${ }^{11}$, we and others have previously noted that CSF P-tau may be increased also as a function of $A \beta$ deposition ${ }^{7,8}$-i.e., provide an indicator of $A \beta$-related tau pathophysiology that anticipates the development of measurable tau tangle pathology. We therefore hypothesized that plasma P-tau217 levels would be related both to $A \beta$ and tau aggregation. Supporting our hypothesis, we found that both $A \beta$ plaques and tau tangles were independently associated with higher plasma P-tau217 levels, both when measured post-mortem by neuropathological quantification (providing the most sensitive indicator of tau tangle deposition) or in vivo by PET imaging (which may not detect tau tangles until there is more substantial and spatially extensive tangle deposition). A $\beta$ plaques and tau tangles also interacted, so that that highest plasma Ptau217 levels were seen in individuals who had high levels of both amyloid plaques and tau tangles. The independent effects of $A \beta$ load and tau tangle load on plasma P-tau217 were significant, with considerable increases in model R2's when combining $A \beta$ and tau predictors compared to when using just one of them alone. Even though the results were mainly similar when using either postmortem neuropathology examination or in vivo PET imaging to detect tau tangles, we found that plasma Ptau217 was more strongly associated with tau tangles than $A \beta$ plaques when using neuropathology examination, which was not the case when using PET (compare Fig. 1A with Fig. 4A). This is probably explained by the fact that tau PET imaging does not reliably detect lower amounts of tau aggregates in the brain, as shown in a recent end-of-life study by Fleischer et al evaluating the diagnostic performance of $18 \mathrm{~F}$-flortaucipir ${ }^{9}$. However, even when using neuropathology examination, A $\beta$ pathology had an 
independent effect on plasma P-tau217 levels, and in cases with more limited tau pathology (mainly restricted to the medial temporal lobe) plasma P-tau217 correlated with A $\beta$ pathology but not tau tangles, although we acknowledge that the methods used for tau quantification in the brain may not be sensitive to the earliest most subtle tau aggregation (Fig. 3). These results are congruent with recent studies showing that both blood and CSF P-tau217 is associated with A $\beta$ PET also in tau PET negative cases 7,12 . Together, these results imply that plasma P-tau217 is not associated with tau tangle pathology independent from $A \beta$ pathology, including primary age-related tauopathy (PART, see Fig. 3B) ${ }^{1}$. However, plasma $P$-tau 217 is associated with $A \beta$ pathology, even during the early stages of the disease (Fig. $3 A$ ), and it is further strongly associated with more widespread tau tangles in cases with $A \beta$ pathology (Fig. $1 \mathrm{C}$ and $4 \mathrm{C}$ ). More research is needed to understand the molecular and cellular mechanisms behind how early $A \beta$ pathology leads to changes in extracellular P-tau217 levels. Overall, the link between $A \beta$ pathology and increased plasma P-tau217 is in line with recent results from cell models ${ }^{13}$ showing that phosphorylation of tau is increased in the presence of $A \beta$.

Plasma P-tau217 also provided statistical mediation (but causal mediation was not tested) for the effects of $A \beta$ load on tau load in both the neuropathology dataset, and for the BioFINDER-2 PET-imaging dataset. Intriguingly, the mediation effect of P-tau217 was especially strong for neuropathological quantification of tangle burden in the neocortex (excluding tangle count densities in entorhinal cortex and hippocampus, Fig. 2C), where plasma P-tau217 explained up to $77 \%$ of the effect of $A \beta$ plaques on tangle burden and the direct effect of $A \beta$ plaques on tangles became non-significant when correcting for $P$ tau217. The in vivo measures with $A \beta$ and global tau PET showed similar results, with considerable mediation for plasma P-tau217 on global tau PET (66\%), but less mediation (29\%) when using tau PET quantified in the entorhinal cortex or hippocampus. This shows that plasma P-tau217 may be especially important for the spread of tau outside of the medial temporal lobe. Isolated medial temporal lobe tangle pathology (which may even appear in the absence of $A \beta$ pathology, including "PART" ${ }^{14}$ ) is not strongly related to increased phosphorylation and/or secretion of tau. When grouping the subjects into three hypothetical "disease stages" (Fig. 6), we found no correlations between plasma P-tau217 and either A $\beta$ PET or tau PET in those that were negative for both $A \beta$ and tau PET (we consider these individuals to not have AD), significant correlations to $A \beta$ PET (but not tau PET) in those who were within the positive range for $A \beta$ PET only (we consider this an early stage of $A D$ ), and significant correlations to both $A \beta P E T$ and tau PET (but strongest with tau PET) in those who were positive for both $A \beta$ and tau PET (we consider this a later stage of $A D$ ). Taken together, these findings are congruent with the hypothesis that $A \beta$ pathology leads to an increased release of soluble P-tau, which in turn is associated with a spread of tau tangle pathology beyond the medial temporal lobe. However, for these tests we note different sensitivities of the biomarkers to detect underlying pathologies could impact their correlations. Figure 8 shows a hypothetical model which integrates the findings from this study and previous studies on biomarkers and development of $A D$. Additional valuable information could come from $A \beta$ overproducing mouse models that do not develop tau tangles, to characterize the extent to which $A \beta$ could promote tau secretion even in the absence of subsequent tau tangle deposition ${ }^{7,15}$. 
Over the last years, there has been an increased interest in anti-tau treatments for $A D^{16}$. Our results suggest that therapies targeting P-tau217 may break the link between buildup of aggregated $A \beta$ and tau, and thereby reduce atrophy and cognitive decline in $A D$ (which are both strongly associated with tau pathology ${ }^{17,18}$ ). At least one tau-directed treatment (using an anti-tau antibody) has been reported to reduce CSF levels of $\mathrm{P}$-tau217 (conference report ${ }^{19}$ ). One intriguing possibility from these findings is that hyperphosphorylated soluble tau may be involved in the pathogenesis of $A D$, and it may be therefore be a suitable treatment target. Further drug development may incorporate plasma P-tau217 data in early stages to select drug candidates most likely to have beneficial effects on the biochemistry of tau in the brain in $A D$.

A limitation of this study is the relatively small dataset in the neuropathological cohort. We used the large BioFINDER-2 cohort to validate the neuropathology findings, but we acknowledge that tau PET has limited sensitivity for early stage tau pathology (with isolated tangle pathology in entorhinal cortex and hippocampus) $)^{9}$. We therefore refrained from doing some of the subgroup analyses in BioFINDER-2 (focusing on MTL tau in individuals with negative global tau).

In summary, plasma P-tau217 is independently associated with both amyloid plaques and tau tangles, including associations with amyloid pathology even in cases with restricted tau tangle pathology, and strong associations with tau tangles in amyloid positive cases.

\section{Methods}

\section{Participants}

Study participants were from two cohorts. First, we included subjects from a biomarker-neuropathology cohort study (the Arizona Study of Aging and Neurodegenerative Disorders/Brain and Body Donation Program), also described previously ${ }^{6}$. Second, we included subjects from the prospective cohort study BioFINDER-2 (clinical trials: NCT03174938), explained previously ${ }^{6}$. From BioFINDER-2, we included all available cognitively unimpaired controls, $A \beta+\mathrm{MCl}$ and $A \beta+A D$ dementia patients (where $A \beta+$ was determined by $A \beta$ PET, explained below). Study demographics are summarized in Table 1 . The data used for this paper partly overlaps with data in a previous publication in diagnostic performance of plasma Ptau $217^{6}$.

\section{Fluid biomarkers}

Plasma P-tau217 was measured using immunoassays at Lilly Research Laboratories, as described before $^{6}$. In the Arizona Study of Aging and Neurodegenerative Disorders/Brain and Body Donation Program, the mean (standard deviation) time between plasma collection and death was $1.2(0.8)$ years. In BioFINDER-2, the mean time between plasma collection and PET imaging was $0.16(0.22)$ years.

\section{Neuropathology}


We included subjects from the biomarker-neuropathology cohort with or without neuropathological evidence of AD. Subjects were excluded that had non-AD tauopathies (including progressive supranuclear palsy $[n=7]$ or corticobasal degeneration $[n=2]$ ) without concomitant AD (since the tangle material in those conditions were thought to potentially confound the associations between plaques, tangles and plasma P-tau217). A $\beta$ plaque and neurofibrillary tangles were graded at standard sites in frontal, temporal, parietal, occipital cortices, the hippocampal CA1 region and entorhinal/transentorhinal cortex, using $80 \mu \mathrm{m}$ Sect. ${ }^{6}$. Plaque densities (including cored, neuritic and diffuse plaques together) were derived using the Campbell-Switzer staining. Neurofibrillary tangle densities were derived using three staining methods (thioflavin S, Campbell-Switzer and Gallyas) on the same sections. The CERAD templates ${ }^{20}$ were used to obtain semi-quantitative regional scores for $A \beta$ and tangle pathology, which were summed to total plaque and tangle scores (range 0-15). All histopathological scoring was blinded to clinical or neuropathological diagnosis (see Supplementary Table 1 for details) and to biomarker levels.

\section{PET imaging}

The procedures of tau PET (using R0948 labeled with radioactive fluorine $\left[{ }^{18} \mathrm{~F}\right]$ ) and AB-PET (using flutemetamol labeled with ${ }^{18} \mathrm{~F}$ ) have been described previously ${ }^{6}$. A $\mathrm{A}$-PET was sampled in a global cortical ROI. When used as a dichotomous variable, A $\beta$-PET > 0.533 SUVR was defined as a positive scan. TauPET was sampled in different ROIs, corresponding to different Braak regions. For most analyses we used a large composite corresponding to Braak regions I-VI, in order to comprehensively capture AD-related brain tau pathology. For some analysis, we used a dichotomous variable for neocortical tau-PETpositivity, using tau-PET uptake in a more limited ROI (corresponding to Braak stages I-IV, where tau-PET $>1.36$ SUVR was defined as a positive $\operatorname{scan}^{10}$ ).

\section{Statistics}

Associations between plasma P-tau217 and measures of $A \beta$ and tau were tested in linear regression models, adjusted for age and sex. Different regression models were compared on the same subjects and outcome, with different sets of predictors (including only $A \beta$ data, only tau data, or both types of data with or without an interaction term). Models were compared using $\mathrm{R}^{2}$ and Akaike Information Criterion (AIC, where a lower value indicates a better fit, corrected for number of predictors). Bootstrap procedures ( $N=1000$ iterations) were used to compare $R^{2}$ between models. Mediation analyses were done to test the relationship between $A \beta$ load, tau load and plasma P-tau217 (using bootstrapped estimates of mediation effects). Statistics were done in $\mathrm{R}$ ( $v$ 4.0.2).

\section{Declarations}

\section{Disclosures}


$\mathrm{OH}$ has acquired research support (for the institution) from AVID Radiopharmaceuticals, Biogen, Eli Lilly, Eisai, GE Healthcare, Pfizer, and Roche. In the past 2 years, he has received consultancy/speaker fees from AC Immune, Alzpath, Biogen, Cerveau and Roche.

\section{Acknowledgements}

Work at the authors' laboratory at Lund University was supported by the Swedish Research Council, the Wallenberg Center for Molecular Medicine, the Knut and Alice Wallenberg foundation, The Medical Faculty at Lund University, Region Skåne, the Marianne and Marcus Wallenberg foundation, the Strategic Research Area MultiPark (Multidisciplinary Research in Parkinson's disease) at Lund University, the Swedish Alzheimer Foundation, the Swedish Brain Foundation, the Swedish Medical Association, the Konung Gustaf V:s och Drottning Victorias Frimurarestiftelse, the Bundy Academy, the Skåne University Hospital Foundation, and the Swedish federal government under the ALF agreement. The Brain and Body Donation Program has been supported by the National Institute of Neurological Disorders and Stroke (U24 NS072026 National Brain and Tissue Resource for Parkinson's Disease and Related Disorders), the National Institute on Aging (P30 AG19610 Arizona Alzheimer's Disease Core Center), the Arizona Department of Health Services (contract 211002, Arizona Alzheimer's Research Center), the Arizona Biomedical Research Commission (contracts 4001, 0011, 05-901, 1001), the Arizona Department of Health Services (Grant No. CTR040636), and the Michael J. Fox Foundation for Parkinson's Research.

\section{References}

1. Sperling, R., Mormino, E. \& Johnson, K. The evolution of preclinical Alzheimer's disease: implications for prevention trials. Neuron 84, 608-622 (2014).

2. Hampel, H. et al. Blood-based biomarkers for Alzheimer disease: mapping the road to the clinic. Nat Rev Neurol (2018) doi:10.1038/s41582-018-0079-7.

3. Olsson, B. et al. CSF and blood biomarkers for the diagnosis of Alzheimer's disease: a systematic review and meta-analysis. Lancet Neuro/ 15, 673-684 (2016).

4. Janelidze, S. et al. Plasma P-tau181 in Alzheimer's disease: relationship to other biomarkers, differential diagnosis, neuropathology and longitudinal progression to Alzheimer's dementia. Nat. Med. 26, 379-386 (2020).

5. Karikari, T. K. et al. Blood phosphorylated tau 181 as a biomarker for Alzheimer's disease: a diagnostic performance and prediction modelling study using data from four prospective cohorts. Lancet Neurol 19, 422-433 (2020).

6. Palmqvist, S. et al. Discriminative Accuracy of Plasma Phospho-tau217 for Alzheimer Disease vs Other Neurodegenerative Disorders. JAMA (2020) doi:10.1001/jama.2020.12134.

7. Mattsson-Carlgren, N. et al. A $\beta$ deposition is associated with increases in soluble and phosphorylated tau that precede a positive Tau PET in Alzheimer's disease. Sci Adv 6, eaaz2387 (2020). 
8. Barthélemy, N. R. et al. A soluble phosphorylated tau signature links tau, amyloid and the evolution of stages of dominantly inherited Alzheimer's disease. Nature Medicine 26, 398-407 (2020).

9. Fleisher, A. S. et al. Positron Emission Tomography Imaging With [18F]flortaucipir and Postmortem Assessment of Alzheimer Disease Neuropathologic Changes. JAMA Neurol 77, 829-839 (2020).

10. Leuzy, A. et al. Diagnostic Performance of R0948 F 18 Tau Positron Emission Tomography in the Differentiation of Alzheimer Disease From Other Neurodegenerative Disorders. JAMA Neurology 77, 1 (2020).

11. Jack, C. R. et al. NIA-AA Research Framework: Toward a biological definition of Alzheimer's disease. Alzheimers Dement 14, 535-562 (2018).

12. Janelidze, S. Associations of Plasma Phospho-Tau217 Levels With Tau Positron Emission Tomography in Early Alzheimer Disease. JAMA Neurology In press,.

13. Kwak, S. S. et al. Amyloid- $\beta 42 / 40$ ratio drives tau pathology in $3 \mathrm{D}$ human neural cell culture models of Alzheimer's disease. Nat Commun 11, 1377 (2020).

14. Crary, J. F. et al. Primary age-related tauopathy (PART): a common pathology associated with human aging. Acta Neuropathol. 128, 755-766 (2014).

15. Maia, L. F. et al. Increased CSF $A \beta$ during the very early phase of cerebral $A \beta$ deposition in mouse models. EMBO Mol Med 7, 895-903 (2015).

16. Bittar, A., Bhatt, N. \& Kayed, R. Advances and considerations in AD tau-targeted immunotherapy. Neurobiol Dis 134, 104707 (2020).

17. Ossenkoppele, R. et al. Associations between tau, $A \beta$, and cortical thickness with cognition in Alzheimer disease. Neurology 92, e601-e612 (2019).

18. Mattsson, N. et al. Predicting diagnosis and cognition with 18F-AV-1451 tau PET and structural MRI in Alzheimer's disease. Alzheimers Dement 15, 570-580 (2019).

19. Active Tau Vaccine: Hints of Slowing Neurodegeneration I ALZFORUM. https://www.alzforum.org/news/conference-coverage/active-tau-vaccine-hints-slowingneurodegeneration.

20. Mirra, S. S. et al. The Consortium to Establish a Registry for Alzheimer's Disease (CERAD). Part II. Standardization of the neuropathologic assessment of Alzheimer's disease. Neurology 41, 479-86 (1991).

21. Hyman, B. T. \& Trojanowski, J. Q. Consensus recommendations for the postmortem diagnosis of Alzheimer disease from the National Institute on Aging and the Reagan Institute Working Group on diagnostic criteria for the neuropathological assessment of Alzheimer disease. J. Neuropathol. Exp. Neurol. 56, 1095-1097 (1997).

\section{Figures}



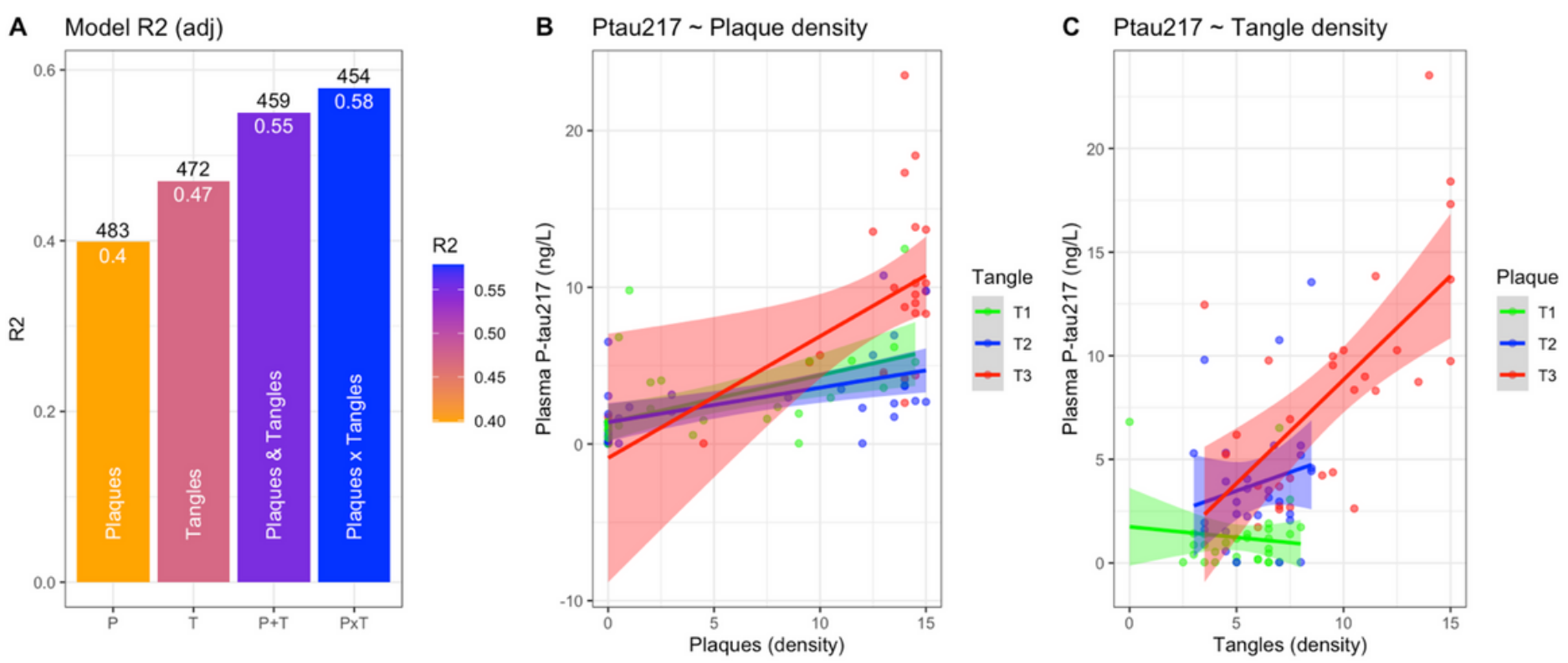

Figure 1

Independent effects of plaque and tangle density on Plasma P-tau217. Panel A shows R2 and AIC for different regression models, using either only amyloid plaque density ("P"), only tangle density ("T"), both plaque and tangle density (" $\mathrm{P}+\mathrm{T}$ "), or both plaque and tangle density including their interaction term ("PxT"). All models included age and sex as covariates. The panel shows adjusted R2, together with AIC (above the bars) for each model. We compared R2 between the models using a bootstrap procedure ( $\mathrm{N}=1000$ iterations), which showed that the $\mathrm{R} 2$ for the "AxT" model was marginally higher than for the "A $+\mathrm{T}$ " model $(\triangle \mathrm{R} 2=0.027,95 \% \mathrm{Cl}-0.0034-0.087)$, and significantly higher than for the " $\mathrm{T}(\mathrm{I}-\mathrm{VI})$ " model $(\triangle \mathrm{R} 2=0.12,95 \% \mathrm{Cl} 0.04-0.24)$ and the "A" model $(\triangle \mathrm{R} 2=0.18,95 \% \mathrm{Cl} 0.055-0.31)$. Panel $\mathrm{B}$ shows associations between plasma P-tau217 and plaque density (stratified by tertiles [T1-3] of tangle density). Panel C shows associations between plasma P-tau217 and tangle count (stratified by tertiles [T1-3] of plaque density). Tertiles were chosen to visualize the data, but the regression models used continuous density data as predictors. 
A

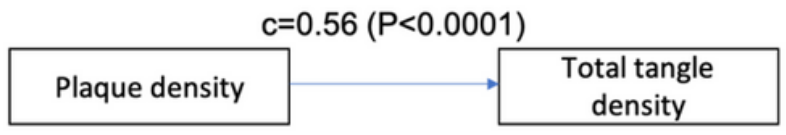

$c-c^{\prime}=0.34[95 \% \mathrm{Cl} 0.15-0.55)(60 \%)$



C

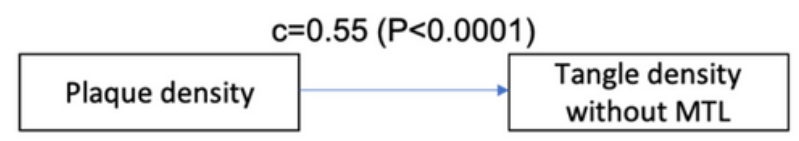

B $\mathrm{C}=0.36(\mathrm{P}=0.00014)$

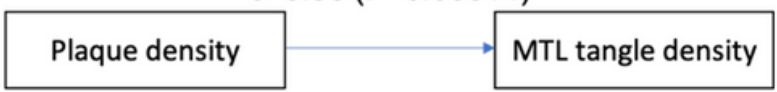

$c-c^{\prime}=0.06[95 \% \mathrm{Cl}-0.06$ to 20.0$)(17 \%)$

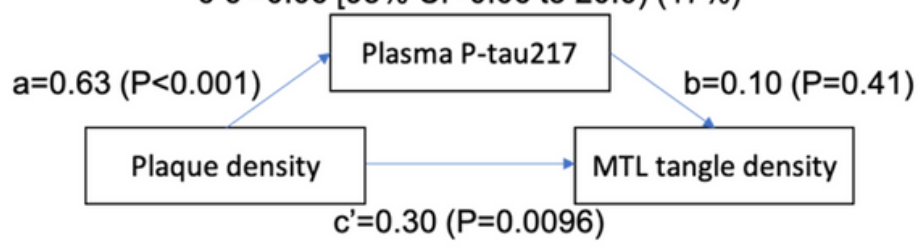

\section{Figure 2}

Plasma P-tau217 mediates effects of plaques on tangles. Three different mediation models are shown, testing plasma P-tau217 as a statistical mediator of plaque density on tangle density. Panel A shows the mediation of total tangle density (significant mediation, $60 \%$ of the direct effect of plaque density on tangle density was explained by plasma P-tau217). Panel B shows the mediation of medial temporal lobe (MTL, entorhinal cortex and hippocampus) tangle density (no mediation). Panel $\mathrm{C}$ shows the mediation of tangle density in all regions except the MTL (strongest mediation, $77 \%$ ).
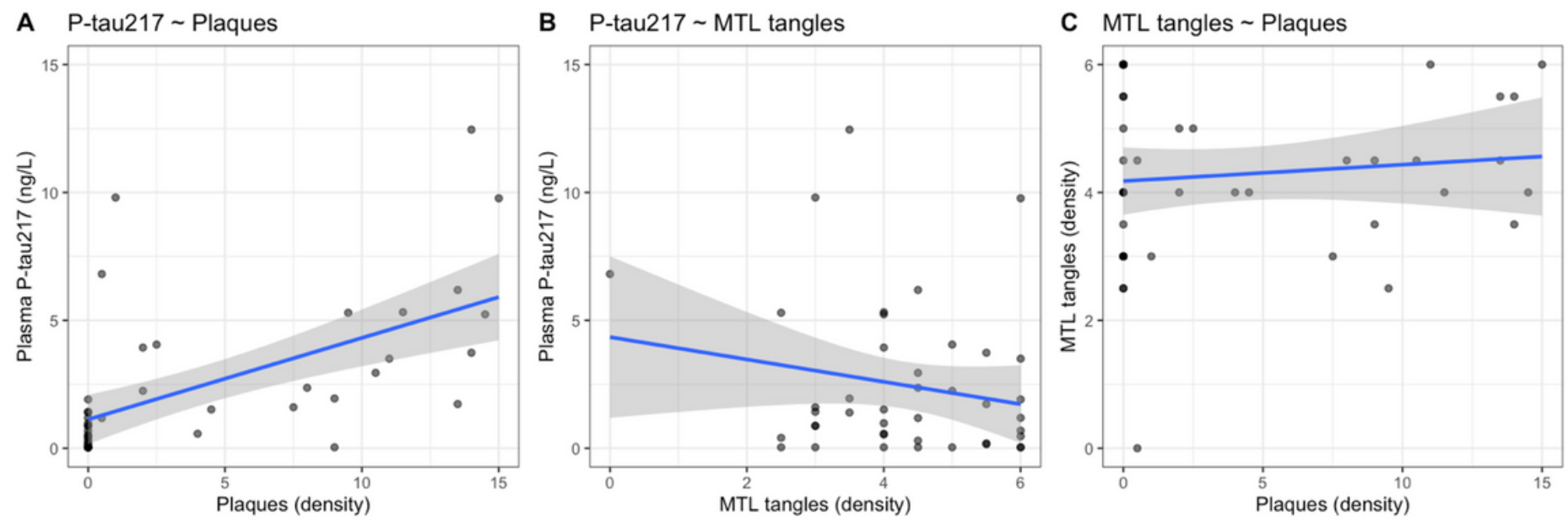

Figure 3 
Associations between plasma P-tau217, A $\beta$ plaque density and medial temporal lobe (MTL) tangle density in people with limited tau pathology. Individuals in the neuropathology cohort without tangles in the parietal or frontal lobe and no more than minimal tangle load in the temporal lobe were included in this analysis $(\mathrm{N}=42)$. MTL tangles were defined as tangles in entorhinal cortex plus hippocampus.
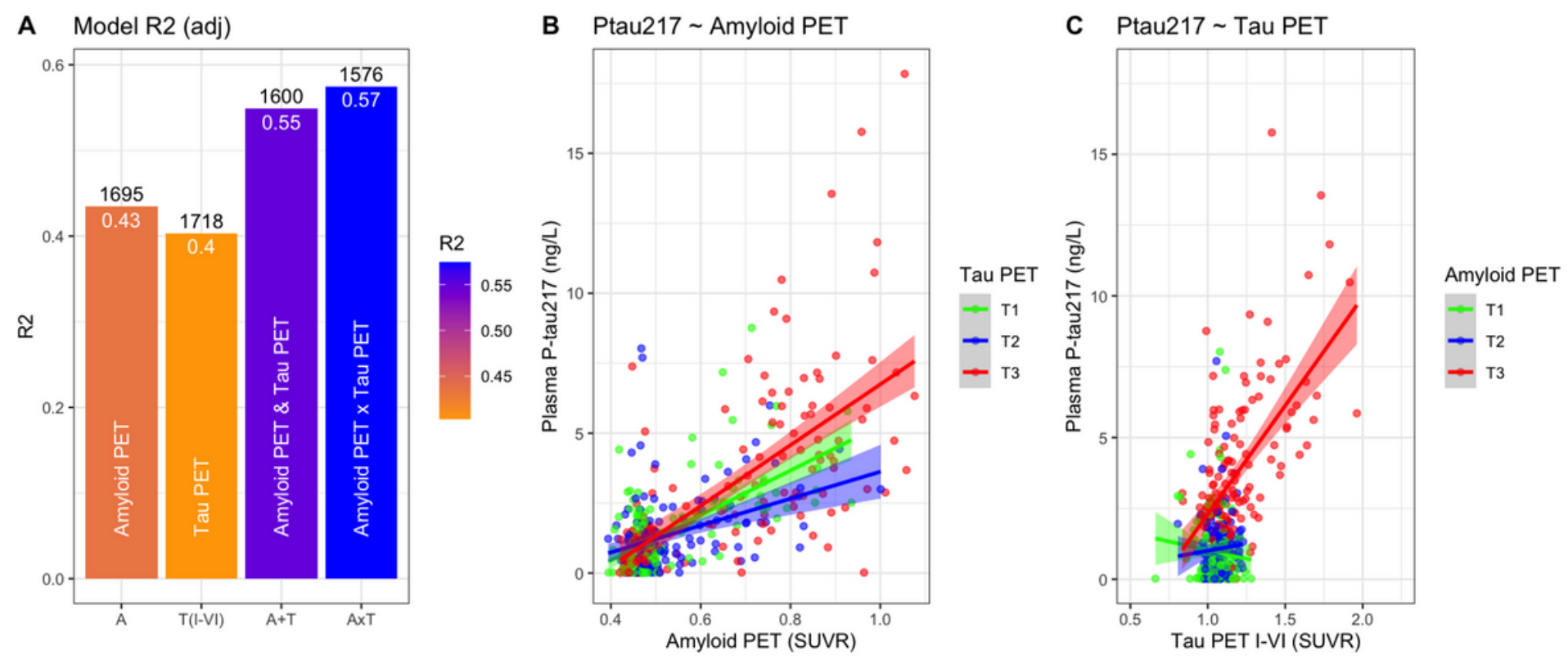

Figure 4

Associations between plasma P-tau217 with A $\beta$ PET and tau PET. Panel A shows R2 and AIC for different regression models, using either only A $\beta$ PET ("A"), only tau PET ("T $(I-V I)$ "), both $A \beta$ and tau PET ("A+T"), or both $A \beta$ and tau PET including their interaction term ("AxT"). All models included age and sex as covariates. The panel shows adjusted R2, together with AIC (above the bars) for each model. We compared $\mathrm{R} 2$ between the models using a bootstrap procedure $(\mathrm{N}=1000)$ iterations, verifying that the $\mathrm{R} 2$ for the "AxT" model was higher than for the "A+T" model ( $\triangle \mathrm{R} 2=0.026,95 \% \mathrm{Cl} 0.010-0.058)$, the "T(I-VI)" model $(\triangle \mathrm{R} 2=0.17,95 \% \mathrm{Cl} 0.11-0.26)$ and the "A" model $(\triangle \mathrm{R} 2=0.14,95 \% \mathrm{Cl} 0.060-0.22)$. Panel $\mathrm{B}$ shows associations between plasma P-tau217 and A $\beta$ PET stratified by tertiles $(T)$ of tau PET (T1: SUVR $\leq 1.05$, T2: $1.05<$ SUVR $\leq 1.11$, T3: $1.11<$ SUVR $\leq 3.03$ ). Panel $C$ shows associations between plasma P-tau217 and tau PET stratified by tertiles (T) of A PET (T1: SUVR $\leq 0.468$, T2: $0.468<$ SUVR $\leq 0.579$, T3: $0.579<$ SUVR $\leq 1.08$ ). For these graphs, tertiles were chosen to visualize the data, but the regression models used continuous PET uptake as predictors. In panel $C$, one outlier was beyond the range of the $x$-axis (Plasma P-tau217 $=17.8 \mathrm{ng} / \mathrm{L}$, Tau PET $=3.03$ SUVR, 3rd Amyloid PET tertile). Note that in other parts of this paper (Figure 6), we used cut-points for AB-PET ( $>0.533$ SUVR) and tau-PET ( $>1.36$ SUVR in a more limited ROI corresponding to Braak stages I-IV) which are different then the levels that define the tertiles. 


\section{Plasma P-tau217 by Amyloid and Tau PET}

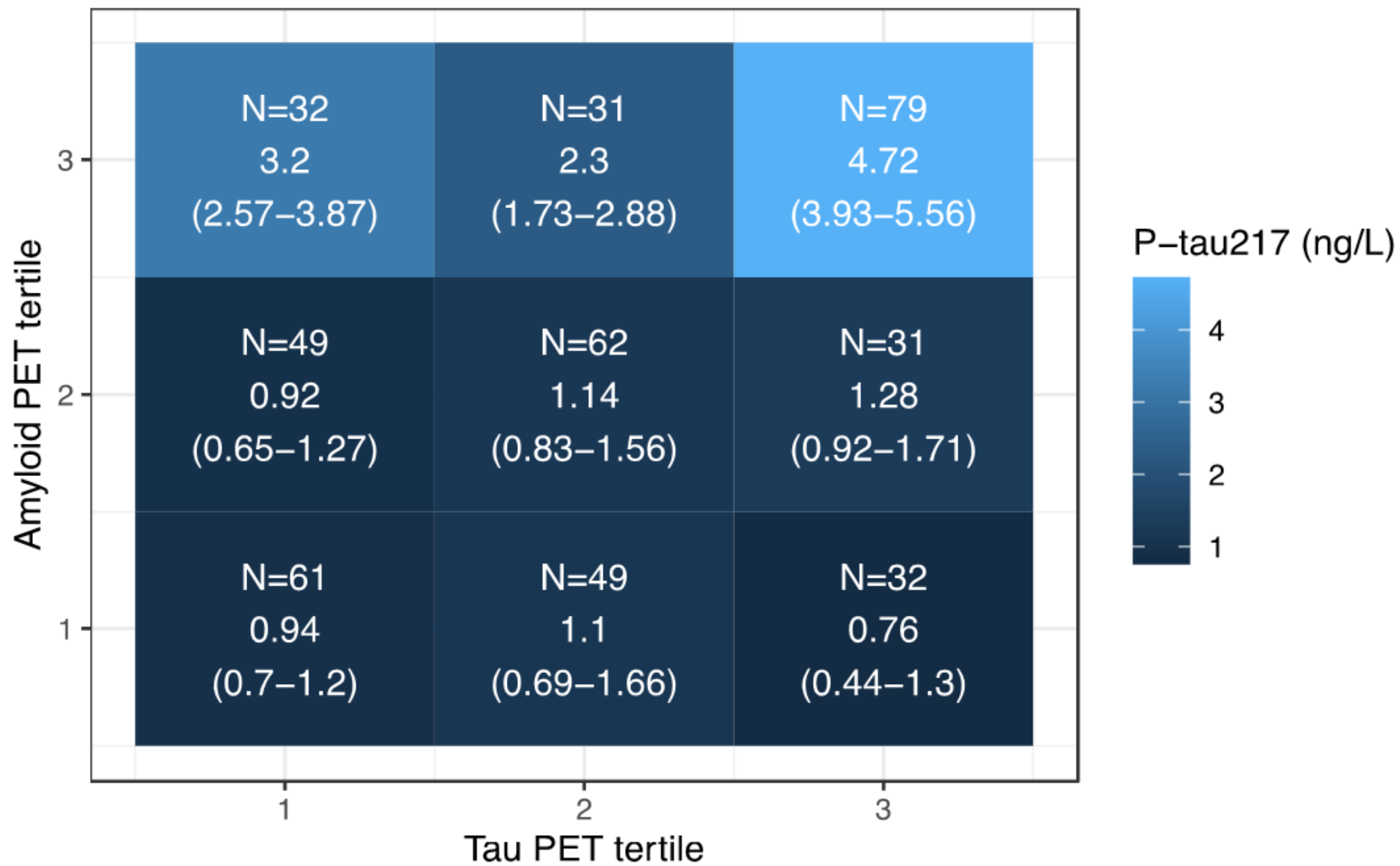

\section{Figure 5}

Plasma P-tau217 by tertiles of A PET and tau PET uptake. The plot shows plasma P-tau217 levels in BioFINDER-2, when grouping subjects by combinations of amyloid and tau PET tertiles. Mean levels are shown together with bootstrapped $95 \%$ confidence intervals ( $N=1000$ iterations). The colors are related to the mean plasma P-tau217 levels in each group. Tertiles defined as follows; tau PET (T1: SUVR $\leq 1.05$, T2: $1.05<$ SUVR $\leq 1.11$, T3: $1.11<$ SUVR $\leq 3.03)$ and A $\beta$ PET (T1: SUVR $\leq 0.468$, T2: $0.468<$ SUVR $\leq$ 0.579 , T3: $0.579<$ SUVR $\leq 1.08$ ). Note that in other parts of this paper (Figure 6), we used cut-points for Aß-PET (> 0.533 SUVR) and tau-PET (> 1.36 SUVR in a more limited ROI corresponding to Braak stages IIV) which were different then the levels that define the tertiles. 

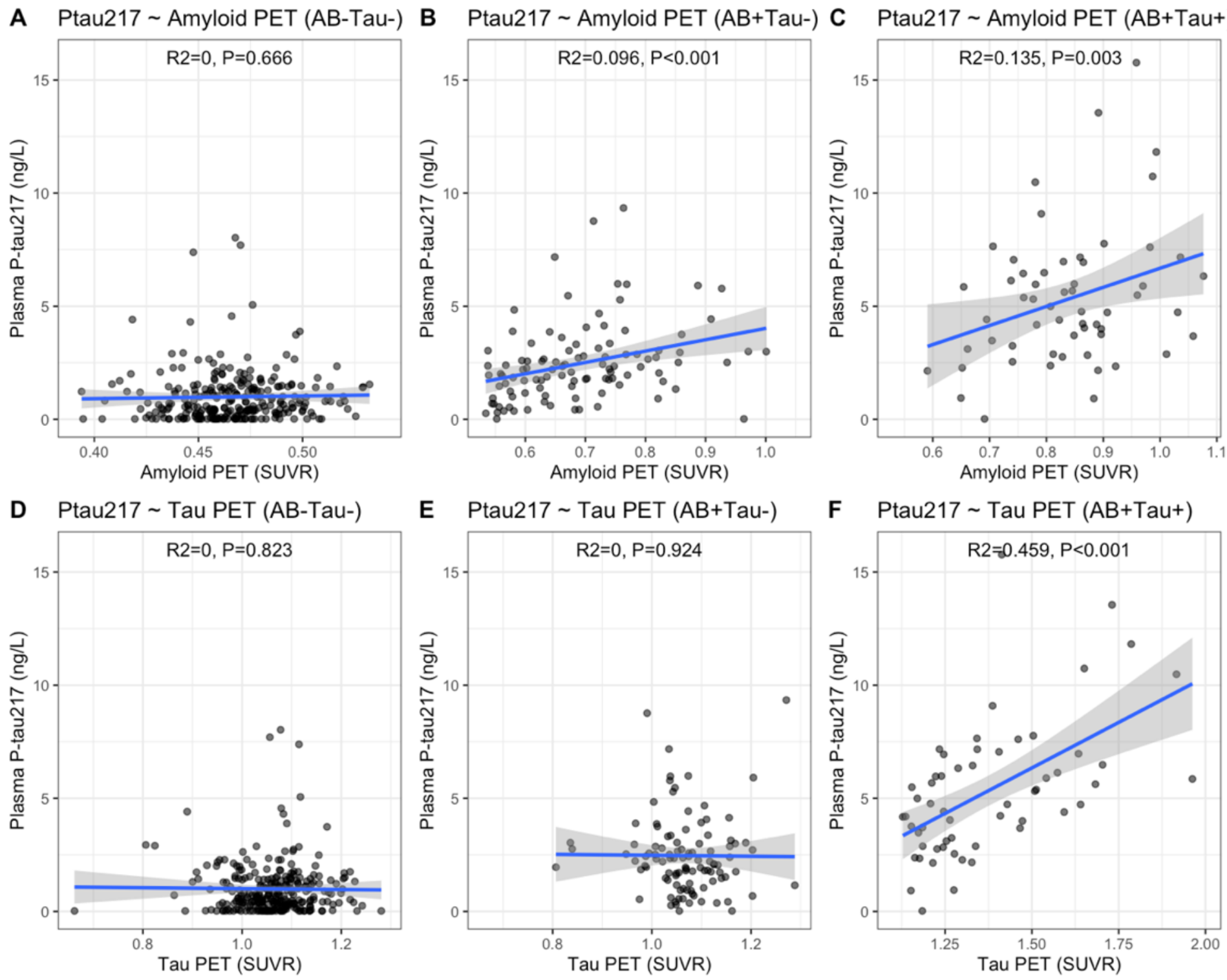

Figure 6

Differential associations with P-tau217 during the course of AD. Associations between plasma P-tau217 and global cortical A $\beta$ PET (panels A-C) and global cortical tau PET (panels D-F) in individuals classified as negative for both $A \beta$ and tau ( $A$ and $D$ ), positive for $A \beta$ only ( $B$ and $E$ ), or positive for both $A \beta$ and tau $(C$ and $F$ ). The plots show R2 and $P$-values from linear regression models. No covariates were included in these models, in order to generate R2-values for the PET measures alone. Cut-points for A $\beta$-PET $(>0.533$ SUVR) and tau-PET (> 1.36 SUVR in a ROI corresponding to Braak stages I-IV) were used to define the groups. Note that these cut-points are different than the ones used to define PET tertiles in other analyses in this paper (Figures 4-5). 
A


B
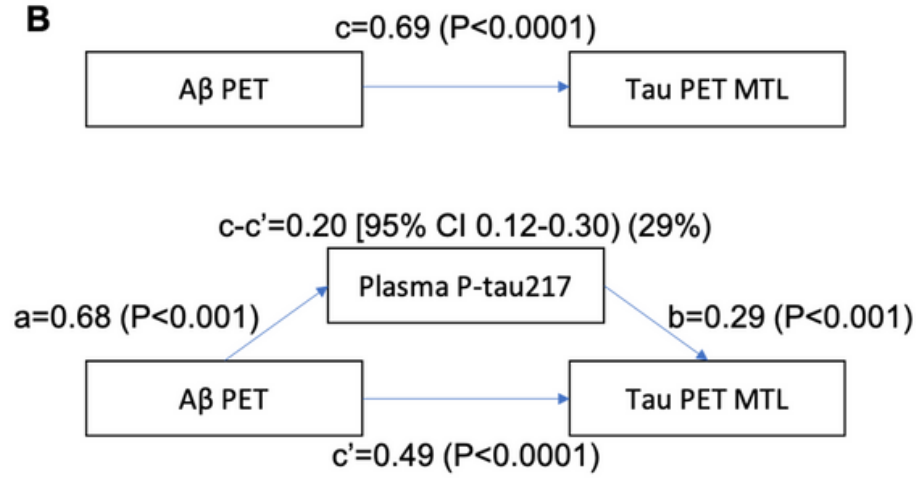

\section{Figure 7}

Mediation analyses in BioFINDER-2. Plasma P-tau217 partly mediated the effect of A PET on tau PET, when using tau PET quantified in Braak stages I-VI (A, "Tau PET global”, $66 \%$ of the direct effect of A PET on tau PET was explained by plasma P-tau217) or in the medial temporal lobe (B, MTL, entorhinal cortex and hippocampus, 29\%).

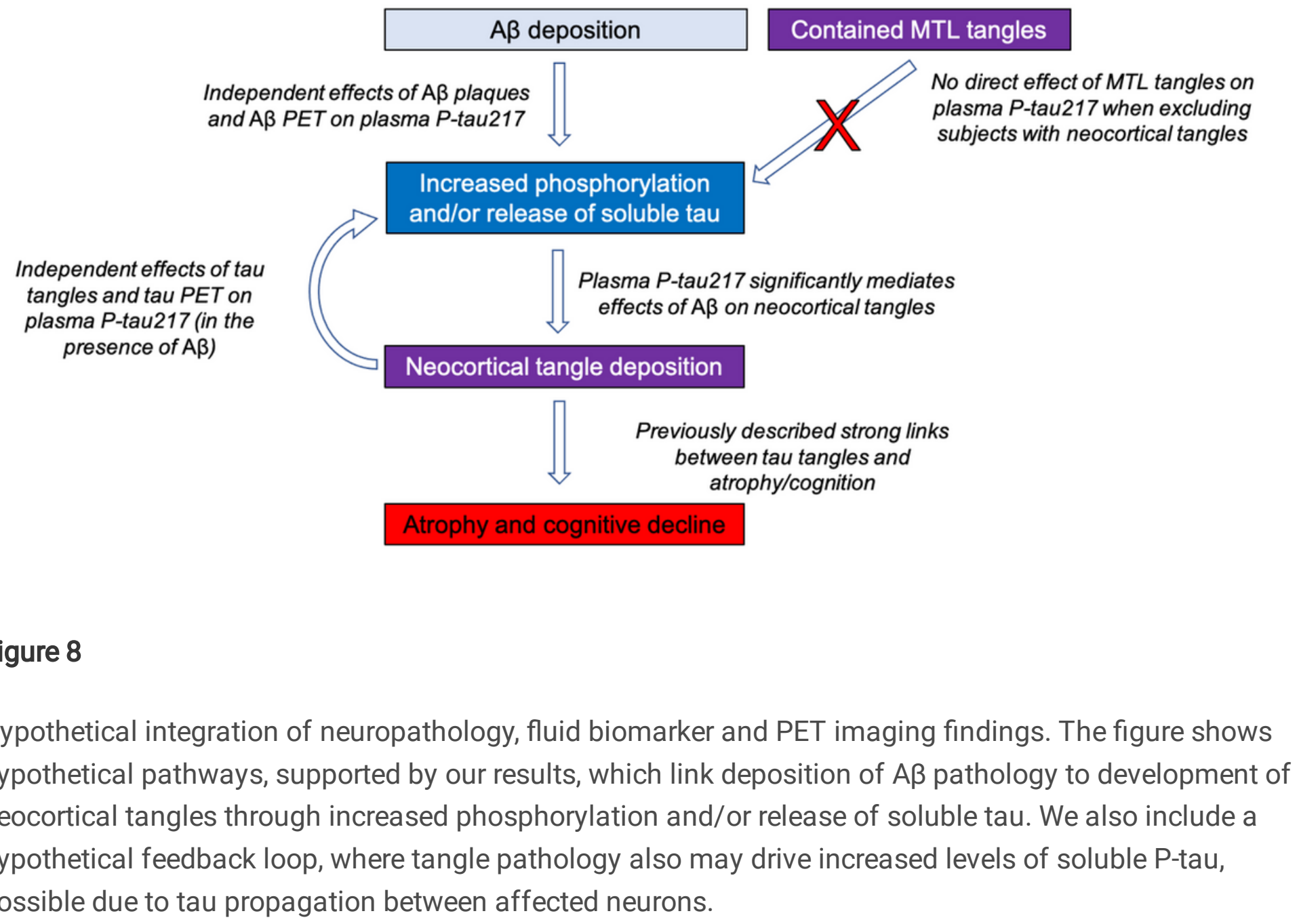




\section{Supplementary Files}

This is a list of supplementary files associated with this preprint. Click to download.

- SupplementaryInformation.docx 